\title{
THE PROBLEM OF LOCALLY- $A$ FUNCTIONS IN A COMMUTATIVE BANACH ALGEBRA $A\left({ }^{1}\right)$
}

\author{
BY \\ RICHARD ARENS
}

1. Introduction. Let $A$ be a commutative Banach algebra with unit. Let $\Delta$ be its space of complex valued homomorphisms. A function $f$ defined on $\Delta$ will be called locally- $A$ if for each point $x \in A$ there is an element $a_{x}$ of $A$ such that $f=\hat{a}_{x}$ in a neighborhood of $x$. The conjecture is that this implies that there is an element $a$ in $A$ such that $f=\hat{a}$. No counter-example of this conjecture is known. On the other hand, there are concrete Banach algebras for which it is not known to hold. For regular Banach algebras, it does of course hold. We give here a sufficient condition, which is satisfied not only by regular algebras, but also by some algebras of analytic functions. We call this condition combinatorial semiregularity. It asks that whenever $f$ is locally- $A$, and the interiors of the set $\left\{f=\hat{a}_{1}\right\}, \cdots,\left\{f=\hat{a}_{n}\right\}$ cover $\Delta$, then there exist regularly closed sets $F_{i}$, and regularly open sets $W_{i}$, such that the $F_{i}$ cover $\Delta$ and

$$
F_{i} \subset W_{i} \subset\left\{f=\hat{a}_{i}\right\} \text {. }
$$

("Regularly" refers to the Stone topology for $\Delta$.)

Now some algebras are not combinatorially semiregular, and we give an example. Further investigation of this example shows that nevertheless it contains all its locally- $A$ functions.

Beyond locally- $A$ functions, one may inquire about functions $f$ given locally by absolutely convergent power series elements (Shilov has considered these). It is shown, on the basis of Cartan's Theorem B, that the problem as to whether every such $f$ is an $\hat{a}$ is equivalent to the vanishing of a certain cohomology group of $\Delta$.

The statement of this theorem requires defining a sheaf $\mathrm{Hol}$ of holomorphic functions in the dual $A^{\prime}$. We then proceed to prove that $H^{q}(\Delta, \mathrm{Hol})$ $=0$ for $q \geqq 1$. The case $q=1$ gives the result mentioned above, which involves a quotient sheaf of Hol.

2. Semiregularity. Let $A$ be a commutative Banach algebra with unit 1 . Let $\Delta(A)=\operatorname{Hom}(A, \mathbf{C})$, that is, the nonzero algebra-homomorphisms of $A$ into the complex numbers C. For $a \in A, \xi \in \Delta(A)$, we define $a_{A}(\xi)$ by $\xi(a)$ (when no ambiguity is possible, we may use " $a$ " for " $a_{A}$ "). We let $S(a)$ be the support of $a_{A}$, i.e., the closure of $\left\{a_{A} \neq 0\right\}$. For a set $E \subset \Delta(A)$ we call the closure of $E$ in the kernel-hull topology [L, p. 60], the regular closure. If $E$

Received by the editors August 7, 1961.

(1) The preparation of this paper was supported in part by the office of Naval Research, Contract NONR 235(60). 
equals its regular closure, we naturally call it regularly closed. Thus $A$ is regular [L, p. 83] precisely when each closed set in $\Delta(A)$ is regularly closed.

We now turn to a concept which we will call regularity. A Banach algebra $A$ shall be called almost regular if it is commutative, has a unit, and if for each $a \in A$, the closed set $S(a)$ is regularly closed. (This is the same as saying that the interior of $\left\{a_{A}=0\right\}$ is regularly open, in an obvious sense.) Regularity obviously implies almost-regularity. The converse is not true. In fact "analytic" algebras have been defined precisely as such for which $S(a)=\Delta(A)$, for $a \neq 0$. (This concept might have been better called "quasi-analyticity.") There are algebras analogous to the algebra of functions continuous on the unit disc, and holomorphic inside, which are not almost regular. We will give examples in $\$ 3$.

We will call a complex-valued function $f$ on $\Delta(A)$ a locally- $A$ function if for each point $\zeta \in \Delta(A)$ there is an $a \in A$ such that $a_{A}=f$ in some neighborhood of $\zeta$. The unsolved problem motivating these investigations is to determine whether there exist any locally- $A$ functions not globally of the form $a_{A}$. If every locally- $A$ function is globally- $A$ (i.e., of the form $a_{A}$ ) we call $A$ a sectionally-complete algebra. To repeat, we know no sectionally incomplete algebra.

It is easy to see that regularity (with its partitions of unity) implies sectional completeness. We want to present another weakened form of regularity which demonstrably implies sectional completeness, since our technique does not enable us to show that almost-regularity will suffice.

A locally- $A$ function is surely continuous. Moreover, the maximummodulus principle of Rossi [R] shows that it necessarily attains its maximum modulus on the Shilov boundary. If $f$ is locally- $A$, and $a \in A$, then $f-a_{A}$ is also a locally- $A$ function. It will, I hope, cause no confusion if we denote it by $f-a .\{f=a\}$ is the set where $f=a_{A}$.

2.1. Definition. We will call $A$ combinatorially semiregular if it is a commutative Banach algebra with unit such that whenever $a_{1}, \cdots, a_{n} \in A$ and $f$, a locally- $A$ function, are given for which the interiors of $\left\{f=a_{i}\right\}$ cover $\Delta(A)$, then there exist regularly closed sets $F_{1}, \cdots, F_{n}$ which cover $\Delta(A)$, and regularly open sets $W_{1}, \cdots, W_{n}$ such that $F_{i} \subset W_{i} \subset\left\{f=a_{i}\right\}$.

Obviously, regular algebras have this property. On the other hand, so do the quasi-analytic ones. For if $A$ is quasi-analytic and $\left\{f=a_{1}\right\},\left\{f=a_{2}\right\}$ have an open nonvoid subset in common, then $a_{1}=a_{2}$. Hence the collection $a_{1}, a_{2}, \cdots, a_{n}$ can be reduced to one in which the interiors $F_{i}$ of the $\left\{f=a_{i}\right\}$ are disjoint and cover the space $\Delta(A)$. Thus the $F_{i}$ are open and closed. Thus they are both regularly open and regularly closed [Sh, '53, A \& C].

It is now easy to prove the following.

2.2. Theorem. Let $A$ be combinatorially semiregular. Then $A$ is sectionally complete. 
Proof. Let $f$ be a locally- $A$ function defined on $\Delta(A)$. By the compactness of $\Delta(A)$ there exist $a_{1}, \cdots, a_{n} \in A$ such that the interiors of the sets $\left\{f=a_{i}\right\}$ cover $\Delta(A)$. By an application of 2.1 we obtain $F_{1}, \cdots, F_{n}, W_{1}, \cdots, W_{n}$ as therein described. Let $E_{k}=W_{k}^{\prime}$ (the complement). Then $E_{k}, F_{k}$ are regularly closed, and so is their union. Let $J$ be the closed ideal of those $a$ for which $a_{A}=0$ on $E_{k} \cup F_{k}$. Then $Q=A / J$ has $\Delta(Q)$ corresponding naturally to $E_{k} \cup F_{k}$. By the result of Shilov already mentioned, there is an element $q \in Q$ such that $g_{Q}=1$ on $F_{k}$, and $=0$ on $E_{k}$. Going back into $A$, we find $u_{k}$ such that $u_{k_{A}}=1$ on $F_{k}$, and $=0$ on $E_{k}$. Let $v_{k}=1-u_{k}$. By setting $1=u_{1}+v_{1}=u_{1}+v_{1}\left(u_{2}+v_{2}\right)$ $=u_{1}+v_{1} u_{2}+v_{1} v_{2}\left(u_{3}+v_{3}\right)=\cdots$ we obtain $1=u_{1}+v_{1} u_{2}+v_{1} v_{2} u_{3}+\cdots+v_{1} v_{2} \cdots$ $v_{n-1} u_{n}+v_{1} v_{2} \cdots v_{n}$. Let us denote the last term here by $v$. Then $v_{A}(\zeta) \neq 0$ only if $u_{i_{A}}(\zeta) \neq 1$ for each $i$, or $\zeta \notin F_{1}$ for each $i$. But this is impossible. Hence $v_{A}=0$. Let $a=u_{1} a_{1}+v_{1} u_{2} a_{2}+\cdots+v_{1} v_{2} \cdots v_{n-1} u_{n} a_{n}$. Here the coefficient of $a_{1}$ is 0 outside of $\left\{f=a_{i}\right\}$. Hence $a_{A}=f$.

This proves 2.2. This proof is pretty trivial, because 2.1 is so strong an assumption. Hence it is perhaps of interest that the case $n=2$ can be treated using only the axiom of almost-regularity.

2.3. Theorem. Let $A$ be almost regular. Let $a_{1}, a_{2} \in A$, and suppose $f$ is such that the interiors of $\left\{f-a_{1}\right\},\left\{f-a_{2}\right\}$ cover $\Delta(A)$. Then $f=a_{A}$ for some $a \in A$.

Proof. Let $d=a_{2}-a_{1}$. Now $S(d)$ is supposed to be regularly closed. Hence it is $\Delta(Q)$ for $Q=A / J$, as before. But $S(d)=S\left(f-a_{1}\right) \cup S\left(f-a_{2}\right)$. These sets are compact and disjoint, and so we can find $q \in Q$ such that $q_{Q}=1$ one one, and $=0$ on the other. Going back into $A$, we find $u$ such that $u_{A}=1$ on $S\left(f-a_{1}\right),=0$ on $S\left(f-a_{2}\right)$. Now let $a=(1-u) a_{1}+u a_{2}$. Now $(1-u)_{A}=0$ when $f \neq a_{1_{\Lambda}}$ and $u_{A}=0$ when $f \neq a_{2_{\Delta}}$. Hence $a_{A}=f$, as desired.

Let $\Sigma(\Delta(A)$, loc. $-A)$ be the algebra of locally- $A$ functions on $\Delta(A)$. We may call it the hull of $A$.

When first considering the problem of sectional completeness, it is natural to speculate what the hull of $A$ could be like, if it were not the same as $A$. For example, if the hull coincides with $\mathfrak{C}(\Delta(A), \mathrm{C})$, what then? This question is easily settled, by the following remark, which is based on the fact that if $\Sigma(\Delta(A)$, loc. $-A)$ is regular, then $A$ is combinatorially semiregular.

2.4. If the hull of $A$ is a regular function algebra on $\Delta(A)$, then the hull of $A$ is just $A$.

If one wants to think of the section problem from the algebraic point of view (rather than the topological one, as we do here) one need not bother with the whole hull of $A$. Just let $\phi$ be one locally- $A$ function, let $A(\phi)$ be the function algebra generated by $A$ and $\phi$ (supposing $A$ to have the sup norm) and then wonder if $A(\phi) \neq A$. (I have heard recently that Dr. G. Stoltzenberg has shown that $A(\phi)$ has the same space of maximal ideals as has $A$.)

Combinatorial semiregularity is ideally suited to generalize a well-known 
proposition concerning the local-belonging of an element $a$ to an ideal $J$ of $A$ $[\mathrm{L}, 85]$. A function $f$ on $\Delta$ is said to belong locally to an ideal $J$ at a point $x \in \Delta$ if there exists an element $b$ in $J$ such that $f=\hat{b}$ in a neighborhood of $x$; and we say $f$ belongs locally to $J$ on $\Delta$ if $f$ belongs locally to $J$ at each $x \in \Delta$.

2.5. Theorem. Let $A$ be combinatorially semiregular. Let $f$ be a complexvalued function on $\Delta$ which belongs locally on $\Delta$ to an ideal $J$ of $A$. Then $f=\hat{a}$ for some $a \in J$.

Proof. We repeat the proof of 2.2 , starting with elements $a_{1}, \cdots, a_{n}$ belonging to $J$, such that the interiors of $\left\{f=a_{1}\right\}, \cdots,\left\{f=a_{n}\right\}$ cover $\Delta(A)$. We take the $\left\{u_{k}\right\}$ and let $a=\sum u_{k} a_{k}$. Evidently $a \in J$ and $f=\hat{a}$, as asserted.

3. Some examples. As we have already said, we know of no commutative Banach algebra $A$ which is not sectionally complete. However, it is perhaps instructive to point out that a related property, which comes to mind in connection with function algebras, is not always possessed by such algebras.

The problem may be formulated as follows. Let $X$ be a compact Hausdorff space. Let $A$ be a uniformly closed subalgebra of $\mathfrak{e}(X, \mathrm{C})$ which contains 1 and separates the points of $X$. Suppose $V_{1}, \cdots, V_{n}$ is an open covering of $X$. Let $f_{1}, \cdots, f_{n}$ be elements of $A$ such that $f_{i}=f_{j}$ on $V_{i} \cap V_{j}$. Does there exist an $f$ in $A$ such that $f=f_{i}$ on $V_{i}(i=1, \cdots, n)$ ? A counterexample is the following. Let $X$ be the union of two disjoint unit circles $X_{1}, X_{2}$ in the complex plane. Let $A$ be the algebra of functions continuous on $X_{1}, X_{2}$ which can be continuously extended into the interior of each circle so as to be holomorphic inside, but for which the mean value on $X_{1}=$ the mean value on $X_{2}$. Now let $V_{k}=X_{k}$, and $f_{k}=k(k=1,2)$. If this were not a counterexample we would have an $f \in A$ such that $f=1$ on $X_{1}, f=2$ on $X_{2}$, contradicting the equality of the two means. The general idea here is to "link" two points of $\Delta\left(A_{1}\right)$ and $\Delta\left(A_{2}\right)$ for $A_{i} \subset \mathfrak{e}\left(X_{i}\right)(i=1,2)$ neither of which lies on its $X_{i}$ as imbedded in $\Delta\left(A_{i}\right)$. In this way, also "inverse-closed" counterexamples can be obtained.

Returning to the original problem of sectional completeness, we remark that counterexamples ought to be sought among those algebras $A$ which are subalgebras of $\mathfrak{e}(\Delta(A))$ which are defined by global properties (and which are not "quasi-analytic"!).

An example of such an algebra is given in [A, '56, §5]. We will sketch the construction briefly. Let $G$ be the group of pairs of integers $(m, n)$. Write $(m, n) \geqq(p, q)$ if $m>p$, or $m=p$ and $n \geqq q$. Let the elements $(m, n)$ of $G$ for which $(m, n) \geqq 0$ form the semigroup $G_{+}$. On the character group $\Gamma$ of $G$ ( $\Gamma$ is identifiable with the torus $\{|z|=|w|=1\} \subset C^{2}$ ) form the function algebra $A_{0}\left(G_{+}\right)$, the uniform closure of the linear combinations of $\left\{z^{m} w^{n}:(m, n) \geqq 0\right\}$.

3.1. For this ordered group $G, A_{0}\left(G_{+}\right)$is not almost regular. As shown in [A, '56], $\Delta\left(A_{0}\right)$ is in $1: 1$ correspondence with the semigroup homomorphisms $\zeta$ of $G_{+}$into the unit disc in C. 
Let $\Omega$ be the class of $\zeta \in \Delta\left(A_{0}\right)$ for which $\zeta((m, n))=0$ for $m \geqq 1$. Then $\Omega$ is precisely the set $\left\{z_{A_{0}}=0\right\}$. Let its interior be called $I$. $I$ does not meet the Shilov boundary ( $\Gamma$ itself, as embedded in $\Delta\left(A_{0}\right)$ ), and hence it cannot contain any nonvoid regularly open set.

3.2. There exist noncombinatorially-semiregular algebras. We first take $A_{0}=A_{0}\left(G_{+}\right)$and form $B=A_{0} \oplus A_{0}$. Then $\Delta(B)$ corresponds naturally to $\Delta\left(A_{0}\right) \times\{0,1\}$. We will call $\Delta\left(A_{0}\right) \times 0$ the lower copy, and $\Delta\left(A_{0}\right) \times 1$ the upper copy, of $\Delta\left(A_{0}\right)$. Each element $f \in B$ is of the type $f_{0} \oplus f_{1}$ where $f((\zeta, 0))=f_{0}(\zeta)$ and $f((\zeta, 1))=f_{1}(\zeta)$, and $f_{0}, f_{1} \in A_{0}\left(G_{+}\right)$. Let $A$ be the subalgebra of $B$ of those $f$ for which $f_{0}$ and $f_{1}$ have the same constant term. (The constant term, or value at the origin, of $f_{0} \in A_{0}$, is its mean value on $\Gamma$.) Then $\Delta(A)$ is obtained by merely "identifying" the origins of the two copies of $\Delta\left(A_{0}\right)$ in $\Delta(B)$. Now to see that $A$ is not combinatorially semiregular. Let $a_{0}=z$ on the lower copy $L$ and $=0$ on the upper copy $U$ of $\Delta\left(A_{0}\right)$. Let $a_{1}=z$ on $U$ and $=0$ on $L$. Then $\left\{a_{0}=0\right\}$ is the union of $U$ and the $\Omega$ on $L$, etc. Hence the interiors of $\left\{a_{0}=0\right\}$, $\left\{a_{1}=0\right\}$ cover $\Delta(A)$. Now suppose we could find regularly open $W, W_{1}$ covering $\Delta(A)$ such that $W \subset\left\{a_{0}=0\right\}, W_{1} \subset\left\{a_{1}=0\right\}$. One of these, say $W$, would contain the coalesced origins 0 . Thus $0 \in W \subset U u(\Omega$ on $L)$, and $0 \notin W^{\prime}$ $\supset(L-\Omega) \supset(\Gamma$-on- $L)$. As remarked earlier, a regularly closed $W^{\prime}$ which contains $\Gamma$ must contain all of the disc.

This shows 3.2.

Finally, we show that

\subsection{Proposition. $A_{0}\left(G_{+}\right)$(the algebra of 3.1) is sectionally complete.}

Proof. We have to recall $\left[\mathrm{A},{ }^{\prime} 56,5.2\right]$ according to which $\Delta\left(A_{0}\left(G_{+}\right)\right)$is homeomorphic to the set $P=\left\{(a, b):(a, b) \in C^{2},|a|,|b| \leqq 1, a=0\right.$ if $\left.|b|<1\right\}$. In this homeomorphism, $z^{m} w^{n}$ is carried into itself (except that its domain is changed to $P$ ). (3.31). On the set $\{|w|<1\} \subset P$ this is holomorphic relative to $w$ because the negative powers of $w$ go only with positive powers of $z$, which are 0 when $|w|<1$. (3.32). On the set $\{|z|<1, w=b,|b|=1\} z^{m} w^{n}$ is holomorphic in $z$. The same is true for all elements of $A_{0}\left(G_{+}\right)$. Let $A(P)$ be the algebra of functions continuous on $P$, and having the analyticity properties just described. Each locally- $A_{0}\left(G_{+}\right)$function is in $A(P)$. Each function in $A(P)$ is uniquely determined by its values on $\Gamma=\{(a, b):|a|=|b|=1\}$. Let now $f \in A(P)$. We shall show that it has the right values on $\Gamma$ for membership in $A_{0}\left(G_{+}\right)$, i.e., that

$$
\int_{0}^{2 \pi} \int_{0}^{2 \pi} f\left(e^{i \theta}, e^{i \phi}\right) e^{i m \theta+i n \phi} d \theta d \phi=0
$$

when $(m, n)>0$. Suppose first $m=0, n \geqq 1$. We integrate first with respect to $\theta$, and get

$$
\int_{0}^{2 \pi} f\left(0, e^{i \phi}\right) e^{i n \phi} d \phi
$$


by 3.32 . And this is 0 by 3.31 . When $m \geqq 1$ we get 0 right away, by 3.32 . So there is a $g \in A_{0}\left(G_{+}\right)$with the same boundary values as $f$. But this means $f=g$ since $f-g \in A(P)$.

This proves 3.3.

4. A sheaf of holomorphic functions on a topological linear space. Let $E$ be a topological linear space. Let $\phi$ be a function defined on, and holomorphic on, some open subset of $C^{m}$. Let $F$ be a linear continuous mapping of $E$ into $\mathrm{C}^{m}$. Then $\phi \circ F$ is defined to be a holomorphic function in $E$, and the class of these functions will be denoted by hol $(E)$. The meaning of sum (or product) of such functions is clear, although the resulting function may have empty domain. It is also easy to see that the resulting function belongs to hol $(E)$.

The functions $f$ in $\operatorname{hol}(E)$ may thus be conceived as follows. Represent $E$ as the direct sum of $E_{0}+E_{1}$ where $E_{1}$ is a finite-dimensional subspace of $E$. Select a function $f_{0}$ defined and holomorphic (in the obvious analytic structure of $\left.E_{1}\right)$ on an open subset $W$ of $E_{1}$. Then let $f\left(x_{0}+x_{1}\right)=f_{0}\left(x_{1}\right)$ for each $x_{0} \in E_{0}, x_{1} \in W$. When dealing with finitely many $f_{i} \in$ hol $(E)$ one can select one complementary pair of subspaces $E_{0}, E_{1}$ to serve for all.

One should note that if $f \in \operatorname{hol}(E)$, and $U$ is an open set, then $\left.f\right|_{U}$ is not necessarily in $\operatorname{hol}(E)$, except in the finite-dimensional case.

No more than in the classical case is it true that if $f, g \in h o l(E)$ agree on some open set, then $f, g$ have some common extension $h \in \operatorname{hol}(E)$. However, one has the following, easily deducible from the finite-dimensional case by the use of a suitable pair of complementary subspaces $E_{0}, E_{1}$.

4.1. Proposition. Let $f, g \in \operatorname{hol}(E)$ and let $U, V$ be open subsets of $E$. Suppose $\left.f\right|_{U_{\cap V}}=\left.g\right|_{U_{\cap} v}$. Then there exists an $h \in \mathrm{hol}(E)$ such that $\left.h\right|_{v}=\left.f\right|_{v}$ and $\left.h\right|_{v}=\left.g\right|_{v}$.

For an infinite subcollection of $f_{i} \in h o l(E)$, the situation is more complicated, and the conclusion of 4.1 cannot be always attained since the resulting function $h$ may not depend merely on finitely many linear functionals.

Let $f$ and $g$ belong to hol $(E)$, and let $x \in E$ belong to both their domains. We write $f \equiv g$ at $x$ if $f-g$ vanishes in a neighborhood of $x$. This is an equivalence relation among those $f \in \operatorname{hol}(E)$ whose domains contain $x$, and we denote the class containing such an $f$, by $[f]_{x}$. The class of all $[f]_{x}$ we call $\operatorname{Hol}(x)$. It is evidently a linear algebra.

We now introduce a topology into $\operatorname{Hol}(E)$, the union of all $\operatorname{Hol}(x), x \in E$. For $f \in \operatorname{hol}(E)$ and $V$ any set in $E$, we define a mapping

$$
[f]_{V}: V \cap \operatorname{domain}(f) \rightarrow \operatorname{Hol}(E)
$$

which sends $x$ into $[f]_{x}$. Denote by $\vartheta$ the class of subsets of $\operatorname{Hol}(E)$ thus obtained, as $V$ ranges over the open sets in $E$. The intersection of two members of $V$ is again a member of $V$, and $v$ covers $E$. Thus there is a topology in $\operatorname{Hol}(E)$ for which $V$ is a basis. The mapping 


$$
\pi: \operatorname{Hol}(E) \rightarrow E
$$

where $\pi\left([f]_{x}\right)=x$, is evidently a local homeomorphism. The space $\operatorname{Hol}(E)$ is in fact a sheaf of linear algebras $[\mathrm{C}(\mathrm{B})]$.

In the finite-dimensional case, a section over an open set $V$ corresponds precisely to an $f \in \operatorname{hol}(E)$ whose domain is that open set, but in the infinitedimensional case this need not hold (see the remark after 4.1). For sections over compact sets, the situation is as follows.

4.2. Theorem. Let $\sigma: K \rightarrow \operatorname{Hol}(E)$ be a continuous mapping such that $\pi(\sigma(x))=x, x \in K$, where $K$ is a compact subset of $E$. Then there exists an $h \in \operatorname{hol}(E)$ and a neighborhood $W$ of $K$ such that $\sigma$ coincides with $[h]_{W}$.

Proof. Let $x \in K$. Then there is an $f_{x} \in \operatorname{hol}(E)$ such that $\sigma$ is given by $\left[f_{x}\right]_{V_{x} \cap K}$ for a neighborhood $V_{x}$ of $x$ in $E$. There are $x_{1}, \cdots, x_{n}$ such that (letting $f_{i}=f_{x_{i}}$, and $\left.V_{i}=V_{x_{i}}\right) V_{1}, \cdots, V_{n}$ cover $K$. Now suppose $x \in \bar{V}_{i} \cap \bar{V}_{j}$ $\cap K$. Then $\sigma(x)=\left[f_{i}\right]_{x}=\left[f_{j}\right]_{x}$, i.e., $f_{i} \equiv f_{j}$ at $x$. This means that $f_{i}$ and $f_{j}$ agree in some neighborhood $W_{x}$ of $x$, i.e., $\left[f_{i}\right]_{W_{x}}=\left[f_{j}\right]_{W_{x}}$ (no restriction to points of $K)$. This means that the set of points $y \in E$ at which $\left[f_{i}\right]_{y} \neq\left[f_{j}\right]_{y}$ is contained in a closed set $T_{i j}$ which is disjoint from $K$. Let $T$ be the union of all these $T_{i j}$, and let $W_{i}=V_{i}-T$. These $W_{i}$ cover $K$. Moreover, $f_{i}\left|w_{i \cap w_{j}}=f_{j}\right|_{w_{i} \cap w_{j}}$. Repeated application of 4.1 yields an $h \in \operatorname{hol}(E)$ such that $\left.f_{i}\right|_{w_{i}}=\left.h\right|_{w_{i}}$, for $i=1, \cdots, n$. This $h$, and the open set $W=W_{1} \cup \cdots \cup W_{n}$ satisfy the conclusion of 4.2 .

5. Cohomology in the space of maximal ideals. Let $A$ be a Banach space and let $A^{\prime}$ be its dual. Let it be given the weak topology $\sigma\left(A^{\prime}, A\right)$. If we let $A^{\prime}$ play the part of $E$ of the preceding section, then the functions in hol $\left(A^{\prime}\right)$ are just those of the form

$$
f(x)=\phi\left(x\left(a_{1}\right), \cdots, x\left(a_{n}\right)\right) \quad\left(x \in A^{\prime}\right)
$$

where $\phi$ is holomorphic in $\mathrm{C}^{n}$, and $a_{1}, \cdots, a_{n}$ are members of $A$. (In fact, the same is true for all topologies lying between the weak topology and the topology first concretely identified in [A, '47], but we will use the weak.)

If $A$ is a commutative Banach algebra with unit, then $\Delta(A)$ (usually abbreviated by $\Delta$, below) is of course a compact subset of $A^{\prime}$. Let $\operatorname{Hol}(\Delta)$ be the sheaf induced on $\Delta$ by $\operatorname{Hol}\left(A^{\prime}\right)$. The elements of this sheaf are those equivalence classes $[f]_{x}$ where $x \in \Delta$. (It has to be borne in mind that $f \equiv g$ at $x$ means $f(y)=g(y)$ for $y$ in a full neighborhood of $x$ in $A^{\prime}$, and that although we may be talking about elements of $\operatorname{Hol}(\Delta)$, we do not have $f$ and $g$ generating the same germ $[f]_{x}$ at $x \in \Delta$ just because they agree on a neighborhood of $x$ relative to $\Delta$.)

The purpose of this section is to establish the following. The general references are $[\mathrm{C}(\mathrm{B})]$ and the Notes to which it refers $[\mathrm{C}(\mathrm{N})]$.

5.1. Theorem. Let $A$ be a commutative Banach algebra with unit, and let 
$\Delta=\Delta(A)$ be the space of complex valued homomorphisms of $A$ as situated in the dual $A^{\prime}$. Then the cohomology groups $H^{q}(\Delta, \operatorname{Hol}(\Delta))=0$ for $q \geqq 1$.

Proof. We proceed indirectly, reducing the question to one about Stein manifolds. Suppose there were a nonzero element of $H^{q}(\Delta, \operatorname{Hol}(\Delta))$. Then we can find a finite covering $\mathcal{u}$ of $\Delta$ by open sets $U_{1}, \cdots, U_{n}$ of $\Delta$ with a nontrivial element $\sigma$ of $H^{q}(N(\mathcal{u}), \operatorname{Hol}(\Delta)$ ) (" $N$ " here means "nerve") which never maps onto 0 in the homomorphism

$$
H^{q}(N(\mathcal{U}), \operatorname{Hol}(\Delta)) \rightarrow H^{q}(N(\mathcal{U}), \operatorname{Hol}(\Delta))
$$

induced by a refinement $v$ of $u$.

This element $\sigma$ defines a section $\sigma_{\mathcal{U}}$ of $\operatorname{Hol}(\Delta)$ over the intersection $U_{i_{0}} \cap \cdots \cap U_{i_{q}}$ of any $q+1$ members of $\mathcal{u}$. We may refine $\mathcal{u}$ by a covering $\mathcal{V}=V_{1}, \cdots, V_{n}$ where $\bar{V}_{i} \subset U_{i}$. Now the section $\sigma_{\mathfrak{U}}$ restricted to $\bar{V}_{i_{0}} \cap \ldots$ $\cap \bar{V}_{i_{q}}$ is by 4.2 represented by a function $h_{i_{0} i_{1}} \ldots q$ defined on, and holomorphic on, an open set $D_{i_{0} i_{1} \cdots i_{q}}$ of the space $A^{\prime}$, where we can require $\Delta \cap D_{i_{0} i_{1}} \cdots i_{q}$ $\subset U_{i_{0}} \cap \cdots \cap U_{i_{q}}$. Therefore, on the set

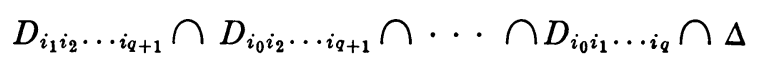

these functions satisfy the relation

$$
h_{i_{1} i_{2} \cdots i_{q+1}}-h_{i_{0} i_{2} \cdots i_{q+1}}+\cdots+(-1)^{q+1} h_{i_{0} \cdots i_{q}}=0
$$

which expresses the fact that the section $\sigma_{\mathcal{U}}$ defines a cocycle in $N(\mathcal{U})$. It is not hard to see that the sets $D_{i_{0} \ldots i_{q}}$ can be so chosen that 5.12 holds on

$$
D_{i_{1} \cdots i_{q+1}} \cap \cdots \cap D_{i_{0} \cdots i}
$$

that is, not merely on $\Delta$. We still have

$$
\bar{V}_{i_{0}} \cap \cdots \cap \bar{V}_{i_{q}} \subset D_{i_{0} \cdots i_{q}} .
$$

We can find a neighborhood $V$ of 0 in $A^{\prime}$ such that

$$
\left(\bar{V}_{i_{0}}+V\right) \cap \cdots \cap\left(\bar{V}_{i q}+V\right) \subset D_{i_{0} \cdots i_{q}}
$$

for every such set of indices $i_{0}, \cdots, i_{q}$. The sets $\bar{V}_{i}+V$ are open in $A^{\prime}$, and their union includes $\Delta$. However, their definition in terms of sets of the form 5.13

$$
\left\{x:\left|x\left(a_{i}\right)-\lambda\right|<\epsilon, i=1, \cdots, r\right\}
$$

might require an infinite collection of elements of $A$. Since, in the weak topology, there is a basis of neighborhoods of the form 5.13 , and since $\Delta$ is compact, we construct a system of open sets $W_{1}, \cdots, W_{u}$ with these properties:

5.14. Each $W_{i}$ is the union of finitely many sets of the type 5.13.

5.15. $W_{i_{0}} \cap \cdots \cap W_{i_{q}} \subset$ domain of $h_{i_{0}} \cdots i_{q}$ for every $(q+1)$-tuple $i_{0}, \cdots, i_{q}$ $\left(1 \leqq i_{j} \leqq u\right)$. 
5.16. On $W_{i_{1} \cdots i_{q+1}} \cap \cdots \cap W_{i_{0} \cdots i_{q}}$ the equation 5.12 holds.

5.17. $\Delta \subset W_{1} \cup \ldots \cup W_{u}$.

We assemble into one linearly independent collection

5.18

$$
a_{1}, \cdots, a_{m}
$$

sufficiently many elements of $A$ to generate linearly all the elements involved in property 5.14 as well as those elements needed to express the $h_{i_{0}} \ldots i_{i}$ in terms of functions holomorphic in finite-dimensional spaces. By setting

$$
F_{m}(x)=\left(x\left(a_{1}\right), \cdots, x\left(a_{m}\right)\right)
$$

we map $A^{\prime}$ into $C^{m}$ linearly and continuously. Moreover

$$
F_{m}\left(W_{i}\right)=\Omega_{i}
$$$$
(i=1, \cdots n)
$$

is open;

5.20

$$
h_{i_{0} \cdots i_{q}}=\phi_{i_{0} \cdots i_{q}} \circ F_{m}
$$

where

5.21

$$
\phi_{i_{0}} \ldots i q \text { is holomorphic on } \Omega_{i_{0}} \cap \cdots \cap \Omega_{i_{q}} \text {; }
$$

and (compare 5.12)

5.22

$$
\phi_{i_{1}} \cdots i_{q+1}-\phi_{i_{0} i_{2}} \cdots i_{q+1}+\cdots=0
$$

on $\Omega_{i_{0}} \cap \cdots \cap \Omega_{i_{q+1}}$;

5.23

$$
F_{m}(\Delta) \subset \Omega=\Omega_{1} \cup \ldots \cup \Omega_{n}
$$

and finally

$$
F_{m}(\Delta) \text { is the joint spectrum } \sigma\left(a_{1}, \cdots, a_{m} ; A\right) .
$$

Therefore $\Omega$ is a neighborhood of $\sigma\left(a_{1}, \cdots, a_{m} ; A\right)$. By [A \& C , 2.3] one can find elements $a_{m+1}, \cdots, a_{n}$ such that, if $C\left(a_{1}, \cdots, a_{n}\right)$ is the algebra generated by $a_{1}, \cdots, a_{n}$, and $p$ is the projection

$$
p: C^{n} \rightarrow C^{m}
$$

wherein $p\left(\lambda_{1}, \cdots, \lambda_{n}\right)=\left(\lambda_{1}, \cdots, \lambda_{m}\right)$, then

$$
\sigma^{\prime} \equiv \sigma\left(a_{1}, \cdots, a_{n} ; C\left(a_{1}, \cdots, a_{n}\right)\right) \subset p^{-1}(\Omega) .
$$

We define $F_{n}$ using $a_{1}, \cdots, a_{n}: F_{n}: A^{\prime} \rightarrow C^{n}$. Then

$$
F_{n}(\Delta) \subset \sigma^{\prime} \text {. }
$$

Now we let $\psi_{i_{0} \cdots i_{q}}=\phi_{i_{0}} \cdots_{i_{q}} \circ p$ and $\Lambda_{i}=p^{-1}\left(\Omega_{i}\right)$. Then, properties 5.20, 5.21 hold when $\phi, \Omega, m$ are replaced by $\psi, \Lambda, n$, and

$$
\sigma^{\prime} \subset \Lambda=\Lambda_{1} \cup \cdots \cup \Lambda_{n} .
$$


As in [A \& C], we now apply Shilov's discovery that $\sigma^{\prime}$ is polynomially convex, and obtain a neighborhood $S$ of $\sigma^{\prime}$,

$$
S=\left\{\left|p_{1}\right|<1\right\} \cap \cdots \cap\left\{\left|p_{N}\right|<1\right\},
$$

where $p_{j}$ are polynomials, and $S \subset \Lambda$. The set $S$ is a Stein manifold [C (B), R], so that Cartan's Theorem B applies (one can also apply Theorem B to $\sigma^{\prime}$ itself) to the effect that $H^{q}(S, \operatorname{Hol}(S))=0(q \geqq 1)$. The functions $\psi_{i_{0}} \ldots i_{q}$ define a $q$-cochain in the nerve of the covering induced by $\Lambda_{1}, \cdots, \Lambda_{n}$, a cochain with coefficients in $\operatorname{Hol}(S)$. From 5.22 as adapted to $n, \Lambda, \psi$ we conclude that this cochain is a cocycle. For some refinement $\left\{M_{\beta}: \beta \in B\right\}$ of the covering $\Lambda_{1}, \cdots, \Lambda_{n}$ we can obtain for each $q$-tuple $\beta_{1}, \cdots, \beta_{q}$, a holomorphic function $\omega_{\beta_{1}} \cdots \beta_{q}$ defined on $M_{\beta_{1}} \cap \cdots \cap M_{\beta_{q}} \equiv M_{\beta_{1}} \cdots \beta_{q}$ such that if $M_{\beta_{j}} \subset \Lambda_{i_{j}}$ for $j=0,1, \cdots, q+1$ then

$$
\psi_{i_{0} \cdots i_{q}}=\omega_{\beta_{1} \cdots \beta_{q}}-\omega_{\beta_{0} \beta_{2} \cdots \beta_{q}}+\cdots
$$

on $M_{\beta_{0}} \cap \cdots \cap M_{\beta_{q+1}}$. Using $F_{n}$ this gives holomorphic functions $\omega \circ F_{n}$ in $A^{\prime}$, and a covering $F_{n}^{-1}\left(M_{\beta}\right)$, which is a refinement of $\mathcal{U}$, and the relation between the $\omega$ and $\psi$ expresses the fact that $\sigma$ is indeed the cohomology class 0 .

This contradiction establishes 5.1.

One application of 5.1 is the next theorem, which has been already presented (Warsaw, Functional Analysis Conference, 1960. It should appear in a publication prepared for that Conference. As I remarked at that time, $\mathrm{H}$. Royden, supposing that I had abandoned the problem, also solved it). These earlier proofs are also based on Cartan's Theorem B.

5.3. Theorem. Let $A$ be as above. Let $G$ be the group of invertible elements, and let $G_{0}$ be the subgroup of exponentials (which is the component of the identity in $G)$. Then $G / G_{0}$ is isomorphic to the Cech cohomology group $H^{1}(\Delta, Z)$, integer coefficients.

Proof. We consider the sheaf $G$-hol $(\Delta)$ of germs of never-vanishing, holomorphic functions. In the last sheaf, the operation is multiplication. One has the exact sequence of homomorphisms

$$
0 \rightarrow Z \stackrel{i}{\rightarrow} \operatorname{Hol}(\Delta) \stackrel{e x}{\rightarrow} G-\operatorname{hol}(\Delta) \rightarrow 0
$$

where $\operatorname{exf}=\exp (2 \pi i f)$, etc. Therefore

$$
0 \rightarrow H^{0}(\Delta, Z) \rightarrow H^{0}(A, \operatorname{Hol}(\Delta)) \rightarrow H^{0}(\Delta, G-\operatorname{hol}(\Delta)) \rightarrow H^{1}(\Delta, Z) \rightarrow 0,
$$

because $H^{1}(\Delta, \operatorname{Hol}(\Delta))=0$, by 5.1 . Thus

$$
H^{1}(\Delta, Z) \approx H^{0}(\Delta, G-\operatorname{hol}(\Delta)) / e x H^{0}(\Delta, \operatorname{Hol}(\Delta)) .
$$

5.32 follows from 5.31 because $\Delta$ is compact. (It might be worth keeping in mind that $A^{\prime}$ is paracompact, and hence exact-sequence arguments apply to all closed subsets.) 
The group $H^{0}(\Delta, G$-hol $(\Delta)$ ), can (by 4.2) be regarded as the multiplicative system of functions holomorphic on a neighborhood of $\Delta$ and not vanishing on $\Delta$, where two functions agreeing on a neighborhood of $\Delta$ are identified. This object we call $H$. It is clearly a group.

We can map $T: H \rightarrow A$, using [A \& C, 6.2] with the result that $x(T(\phi))$ $=\phi(x)$ for each $x \in \Delta$. Thus $T(H) \subset G$. Whether $T$ is multiplicative we do not know, but it does not matter. Following $T$ by the canonic map $G \rightarrow G / G_{0}$ we get

$$
T_{1}: H \rightarrow G / G_{0} .
$$

$T_{1}$ is a homomorphism. Indeed $T(\phi \psi) T(\phi)^{-1}-1$ belongs to the radical of $A$ and can therefore be written in the form $e^{r}-1$ [A \& C, 7.3], where $r$ is in (the radical of) $A$. This shows that $T_{1}$ is a homomorphism. Now to show that $T_{1}$ is $1: 1$. Let $T(\phi)=e^{a}$. Let $\psi=\exp (-\dot{a})$ where $\dot{a}(x)=x(a), x \in A^{\prime}$. Then the function $\phi \psi$ has the value 1 on $\Delta$ and hence is of the form $e^{\omega}$ in some neighborhood of $\Delta$. The same is true for $\phi$. Thus $T_{1}$ is $1: 1$. Finally, $T_{1}$ is onto. For let $a \in G$ be given. Let $\phi=\dot{a}(\dot{a}(x)=x(a))$. Then $\phi \in H$ and $T_{1}(\phi)$ is easily seen to be $a G_{0}$.

This proves 5.3.

Actually, there is a homomorphism of $H^{0}(\Delta, \operatorname{Hol}(\Delta))$ onto $A$ which sends $\dot{a} \rightarrow a$ for each $a \in A$. This follows from [A, '61].

We now return to a reformulation of the problem of the locally- $A$ functions.

6. Locally- $A$ functions and cohomology. Let $A, A^{\prime}, \Delta=\Delta(A)$ be as in the previous sections. Let $\mathcal{Z}(\Delta)$ be the subsheaf of $\operatorname{Hol}(\Delta)$ consisting of those germs $\xi$ which contain functions $f$ vanishing on a relative neighborhood of $\pi(\xi) \in \Delta$. The sequence of homomorphisms

$$
0 \rightarrow \mathrm{Z}(\Delta) \rightarrow \operatorname{Hol}(\Delta) \rightarrow \operatorname{Hol}(\Delta) / \mathrm{Z}(\Delta) \rightarrow 0
$$

is exact, and so we have (using 5.1 to get the final 0 )

$$
0 \rightarrow H^{0}(\Delta, \mathrm{Z}(\Delta)) \stackrel{i}{\rightarrow} H^{0}(\Delta, \operatorname{Hol}(\Delta)) \stackrel{q}{\rightarrow} H^{0}(\Delta, \operatorname{Hol}(\Delta) / \mathcal{Z}(\Delta))
$$

$$
\stackrel{r}{\rightarrow} H^{1}(\Delta, \mathrm{Z}(\Delta)) \rightarrow 0 \text {. }
$$

6.3. Proposition. A locally-A function is essentially an element of

$$
\tilde{A}=H^{0}(\Delta, \operatorname{Hol}(\Delta) / \mathrm{Z}(\Delta)) \text {. }
$$

Proof. Let $\phi$ be the locally- $A$ function. Then there exist elements $a_{1}, \cdots$, $a_{n}$ of $A$, and neighborhoods $U_{1}, \cdots, U_{n}$ open in $A^{\prime}$ such that $\phi(x)=x(a)$ for $x \in U_{i} \cap \Delta$. Naturally $\dot{a}_{i}=\dot{a}_{j}$ on $U_{i} \cap U_{j} \cap \Delta$. Thus $\left[a_{i}\right]_{x}-\left[a_{j}\right]_{x} \in \mathbb{Z}(\Delta)$ for each $x \in U_{i} \cap U_{j} \cap \Delta$. This proves 6.3.

Now suppose $H^{1}(\Delta, \mathrm{Z}(\Delta))=0$. Then, by 6.2 , the mapping $q$ from 
$H^{0}(\Delta, \operatorname{Hol}(\Delta))$ to $\tilde{A}$ is onto. This means (by 4.2) that there is a holomorphic $f$ defined on a neighborhood of $\Delta$ such that $[f]_{x}-\left[\dot{a}_{i}\right]_{x} \in Z(\Delta)$ for $x \in U_{i} \cap \Delta$. Therefore, $f(x)=\phi(x)$ for $x \in \Delta$. Now $f$ gives rise to an element $a \in A$ for which $f(x)=x(a)$ (we refer to [A \& C, 6.2] as in the proof of 5.3). This proves the following.

6.4. Theorem. If $H^{1}(\Delta, \mathrm{Z}(\Delta))=0$ then $A$ is sectionally complete.

Let us consider the set $\tilde{A}$.

6.5. Proposition. $\tilde{A}$ is in 1:1 correspondence with the class of functions $f$ defined on $\Delta$ for which, for each $x_{0} \in \Delta$, there exist elements $a_{1}, \cdots, a_{n}$, and $a$ holomorphic function $\phi$ in $C^{n}$ such that

$$
\phi\left(x\left(a_{1}\right), \cdots, x\left(a_{n}\right)\right)
$$

is defined for all $x$ in a neighborhood of $x_{0}$ in $\Delta$, and equals $f(x)$.

This proposition is obvious, and we omit the proof. Functions of the type described in 6.5 we shall call $\operatorname{locally}-\operatorname{Hol}(A)$. Such functions were considered by Shilov [Sh] who asserted that each locally-Hol $(A)$ function $f$ had the form $\hat{a}\left(=\left.\dot{a}\right|_{\Delta}\right)$ for some $a \in A$. (Such a result would of course go well beyond solving the considerably older problem about locally- $A$ functions.) For all we know this may also be true. However, we can at most relate this problem to another, as follows.

6.6. TheOREM. Each locally-Hol $(A)$ function $f$ is of the form a for some $a \in A$ if and only if $H^{1}(\Delta, \mathcal{Z}(\Delta))=0$.

Proof. The "if" part is just a slight generalization of 6.4 , and thus does not involve 5.1. We now consider the "only if." If a locally-Hol $(A)$ function $f$ comes from an element $a \in A$, then the element of $\tilde{A}$ corresponding to $f$ is the image under $q$ of the section of $\operatorname{Hol}(\Delta)$ defined by $\dot{a}$. Thus "only if" implies that $q$ in 6.2 has range $\tilde{A}$. That being so, the mapping $r$ must annihilate $\tilde{A}$. But (from 5.1), $r(\tilde{A})=H^{1}(\Delta, \mathrm{Z}(\Delta))$.

7. Appendix. We wish to correct [A, '56] as follows.

To the fourth sentence on p. 504 add the alternative "or have arbitrarily small numerical values on the imaginary axis." To the first sentence of 2.7 on p. 504 add the condition "and suppose inf - $<<<\infty|f(i v)|>0$."

Dr. D. A. Edwards, On absolutely convergent Dirichlet series, Proc. Amer. Math. Soc. 8 (1957), 1067-1074 shows essentially that the condition (which is obviously necessary) does not follow from the remaining conditions. In any case, the omission was an oversight.

\section{BIBLIOGRAPHY}

A, '47. R. Arens, Duality in linear spaces, Duke Math. J. 14 (1947), 787-794.

A, '56. - A Banach-algebra generalization of conformal mappings of the disc, Trans. Amer. Math. Soc. 81 (1956), 501-513. 
A, '61. The analytic-functional calculus in commutative topological algebras, Pacific J. Math. 11 (1961), 405-429.

A \& C. R. Arens and A. P. Calderon, Analytic functions of several Banach algebra elements, Ann. of Math. (2) 62 (1955), 204-216.

C (B). H. Cartan, Variêtés analytiques complexes et cohomologie, Colloque sur les fonctions de plusieurs variables, Bruxelles, 1953, pp. 41-55. (Math. Rev. 16, 234.)

C (N). - Seminaire Ecole Normale Superieure, 1951-1952, Chapters XIX-XX.

L. L. H. Loomis, Abstract harmonic analysis, Van Nostrand, New York, 1953.

R. Hugo Rossi, The local maximum-modulus principle, Ann. of Math. (2) 72 (1960), 1-11.

Sh, '53. G. E. Shilov, On the decomposition of a commutative normed ring into a direct sum of ideals, Mat. Sb. N.S. 32 (1953), 353-364 (in Russian).

Sh, '60. - Analytic functions in a normed ring, Uspehi Mat. Nauk 15 (1960), no. 3 (93), 181-183.

University of California,

Los Angeles, California 